\title{
Simulation on binding efficiency of immunoassay for a biosensor with applying electrothermal effect
}

\author{
Kuan-Rong Huang, Jeng-Shian Chang, ${ }^{\text {a) }}$ Sheng D. Chao, ${ }^{\text {b) }}$ Kuang-Chong Wu, \\ Chih-Kai Yang, Cheng-Yu Lai, and Shyh-Haur Chen \\ Institute of Applied Mechanics, National Taiwan University, Taipei 106, Taiwan, Republic of China
}

(Received 26 March 2008; accepted 24 July 2008; published online 25 September 2008)

\begin{abstract}
The working principle of immunoassays is based on the specific binding reaction of an analyte-ligand protein pair in physiological environments. However, for a diffusion-limited protein, the diffusion boundary layer of the analyte on the reaction surface of a biosensor would hinder the binding reaction from association and dissociation. The formation of such association and dissociation layers thus limits the response time and the overall performance of a biosensor. In this work we have performed a two-dimensional full time scale finite element simulation on the binding reaction kinetics of two commonly used proteins, C-reactive protein (CRP) and immunoglobulin G $(\operatorname{IgG})$. By applying a nonuniform ac electric field to the flow microchannel of the biosensor, the electrothermal force can be generated to induce a pair of vortices to stir the flow field. With the aid of the vortices and a suitable choice of the location of the biosensor, the fluids flowing over the reacting surface can be accelerated fast enough to depress efficiently the growth of the diffusion boundary layer on the reaction surface, and enhance the association or dissociation of analyte-ligand complex. The interference patterns of the flow field due to the existence of the sensor at different locations of the microchannel could cause different degrees of enhancement to the association and the dissociation. By changing the location of the sensor the largest enhancement is found at the position near the negative electrode. For the configuration of the microchannel we studied, the initial slope of the curve of the analyte-ligand complex versus time can be raised up to 5.17 for CRP and 1.93 for IgG in association, and 3.74 for CRP and 1.28 for IgG in dissociation, respectively, under the applied ac field $15 \mathrm{~V}_{\text {rms }}$ peak-to-peak and operating frequency $100 \mathrm{kHz}$. At this optimal sensor location, we also studied the effect of various settings of temperature boundary conditions on the top and bottom walls, including the two limiting cases, namely, constant temperature and thermal insulation on both walls. We show that varying the temperature boundary conditions can cause an essential effect on the enhancement of the binding reaction and can be employed to find an optimal binding enhancement. Utilizing these simulation results, an improved design incorporating a pair of electrodes and a neck region near the reaction surface is demonstrated. The sensor is fixed to locate at the middle of the bottom side. With the existence of the stirring flow field, the association rate of the $30 \mu \mathrm{m}$ neck is 2.73 times faster than that of the original channel with no neck. (C) 2008 American Institute of Physics. [DOI: 10.1063/1.2981195]
\end{abstract}

\section{INTRODUCTION}

Recent rapid advances in micro-/nanotechnologies have given impetus to the development and design of microfluidic biosensors for health-care applications, such as immunoassays. The possibility of fabricating and integrating microsize biosensors of multiple functions has led to the idea of performing real-time monitoring or diagnostics on a portable laboratory on a chip. The three most commonly used devices in detecting biomolecules are the microcantilever beam based biosensor, the surface plasmon resonance (SPR) sensor, and the quartz crystal microbalance (QCM) sensor. Although the mechanisms of detection are different, they all involve the same kinetics of specific binding of analytes, such as C-reactive protein ${ }^{1}$ (CRP) and immunoglobulin G $(\operatorname{IgG})$, and immobilized ligands, such as anti-CRP and antiIgG. The concentration of the formed analyte-ligand com-

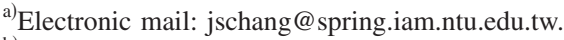

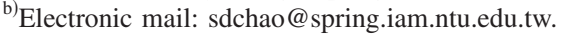

plex during the chemical binding is the major quantity correlating with the measured data from various sensors, and reflects the concentration of analytes in the flow, which is the physical quantity most concerned in clinic applications.

The specific recognition of analytes and immobilized ligands occurs at the reaction surface of a biosensor, which is a solid-liquid interface. The reaction kinetics can be described as a two-step process. ${ }^{2}$

(1) Mass-transport process: the analyte is transported by diffusion from the bulk solution toward the reaction surface $[A]_{\text {bulk }} \rightleftarrows[A]_{\text {surface }}$.

(2) Chemical reaction process: the binding of the protein pair takes place

$[A]_{\text {surface }}+[B] \underset{k_{d}}{\stackrel{k_{a}}{\rightleftarrows}}[A B]$,

where the concentration $[A]_{\text {bulk }}$ is for the analyte in the bulk, $[A]_{\text {surface }}$ is for the analyte at the reaction surface, 
$[B]$ is for the ligand, and $[A B]$ is for the analyte-ligand complex, respectively. Here $k_{a}$ is the association rate constant, and $k_{d}$ is the dissociation rate constant.

When the flow rate (hence the convective velocity for analyte) in a biosensor is fixed, the required experiment time of a specific biomolecular recognition usually depends on its Damköhler number $\left(D_{a}\right.$ number), which is a dimensionless parameter to measure whether a reaction is diffusion limited or reaction rate limited. The $D_{a}$ number is the ratio of reaction velocity (i.e., product of the association rate constant and the initial concentration of the ligand) to diffusion velocity (i.e., ratio of the diffusion coefficient of the analyte in the buffer flow to the height of microchannel). ${ }^{3}$ When the $D_{a}$ number is greater than unity, the whole reaction is restrained by diffusion, i.e., diffusion transport limited.

The development of immunoassays is to place emphasis on the high sensitivity and the real-time detecting ability for different mechanism-based biosensors. When the analyte takes a longer time to transport by convection and diffusion to the reaction surface than chemical reaction, the whole reaction is restrained by mass-transport limitation and it usually causes the formation of a diffusion boundary layer. ${ }^{4}$ The formation of such layers would limit the response time and the overall performance of the biosensor. In practice, it often takes hours to complete a detection cycle, which is the main technical problem to be solved. Sigurdson et al. ${ }^{5}$ found that the electrothermal microstirring effect in a microchannel is useful to enhance the binding efficiency for diffusion-limited molecules (large $D_{a}$ proteins). It is promising in our previous study $^{6}$ that several fabrication parameters can be tuned to improve the performance of a biosensor.

Microelectrode structures are commonly used in ac electrokinetics to generate the high strength ac electric field, which is required to move suspended particles in liquid. Particles with a wide range of sizes have been dielectrophoretically manipulated in this manner, from cells $(\sim 10 \mu \mathrm{m})$ and bacteria down to viruses $(\sim 100 \mathrm{~nm})$ and protein molecules. ${ }^{8,9}$ Ac electrokinetics can be classified into three kinds of force: dielectrophoresis, electrothermal force, and electro-osmosis. ${ }^{10}$ While a nonuniform ac electric field can move suspended particles using dielectrophoretic forces, it can also move the fluid through the electrothermal effect or ac electro-osmosis. ${ }^{11-13}$ Ac electro-osmosis is only influential at the frequency below $10 \mathrm{kHz}$ and is likely to induce hydrolysis at the surface of microelectrodes at low frequency. ${ }^{14}$ Dielectrophoresis does not significantly affect the motion of the particle at the submicrometer scale. In contrast, the electrothermal effect is dominant in the bulk fluid at higher frequency. ${ }^{15}$ Therefore, only the electrothermal force is discussed for the simulation of protein immunoassay in our study.

In this paper we simulate a two-dimensional (2D) full time scale process of association and dissociation in a biosensor immunoassay with the samples of CRP pairs and IgG pairs to predict the surface concentration of the analyteligand complex versus time relationship. The induced electrothermal force by the ac electric field can cause a vortex field which will reduce the thickness of the diffusion bound- ary layer and significantly increase the reaction rate to accelerate both the association and dissociation processes. Interference patterns of the vortex field due to the existence of the sensor at various locations in the microchannel could cause different degrees of enhancement of association and dissociation and will be discussed in this work.

Due to the nature of the detecting mechanism, many biosensors (such as SPR and QCM) place the reacting surface in the bottom position of the microchannel. As an illustrating example of designing a biosensor with better performance, we propose a neck design of the microchannel in addition to including the electrothermal effect by introducing a pair of electrodes on the top position of the microchannel opposite to the reacting surface. The neck design of the microchannel reduces the distance of diffusion of analyte in the bulk solution to the reaction surface and also raises the velocity of the solution flowing over the reaction surface. Combining this passive mechanism by changing geometries with the active mechanism provided by the electrothermal effect, we will show that the rate of association and dissociation can be effectively raised.

\section{THEORY}

In this section the equations governing the electrothermal force, the electric field, the temperature field, the flow field, the concentration field, and the biochemical reaction are described. Detailed geometry, flow properties, binding constants, electrodes, and initial and boundary conditions that are required for simulation are described in Sect III.

\section{A. Electrothermal force}

Temperature gradients occur as a result of Joule heating due to the nonuniform ac electric field. The gradient of temperature $T$ in the liquid causes inhomogeneities of the permittivity $\varepsilon$ and the conductivity $\sigma$ of the medium, which in turn gives rise to the forces causing fluid motion. The body force $\overrightarrow{F_{E}}$ is given by ${ }^{10}$

$$
\overrightarrow{F_{E}}=-\frac{1}{2}\left[\left(\frac{\nabla \sigma}{\sigma}-\frac{\nabla \varepsilon}{\varepsilon}\right) \cdot \vec{E} \frac{\varepsilon \vec{E}}{1+(\omega \tau)^{2}}+\frac{1}{2}|\vec{E}|^{2} \nabla \varepsilon\right],
$$

where $\tau=\varepsilon / \sigma$ is the charge relaxation time, and $\omega$ is angular frequency of the electric field $\vec{E}$, respectively. The local variations in temperature change the gradients of conductivity and permittivity

$$
\begin{aligned}
& \nabla \varepsilon=(\partial \varepsilon / \partial T) \nabla T, \\
& \nabla \sigma=(\partial \sigma / \partial T) \nabla T .
\end{aligned}
$$

The force induced by the permittivity gradient is the dielectric force. The force induced by the conductivity gradient is the Coulomb force. If $\omega \ll \sigma / \varepsilon$, the force is dominated by the Coulomb force. If $\omega \gg \sigma / \varepsilon$, the force is dominated by the dielectric force. For water, $1 / \varepsilon(\partial \varepsilon / \partial T)=$ $-0.4 \%, \quad 1 / \sigma(\partial \sigma / \partial T)=2 \%$ per degree Kelvin, ${ }^{16}$ Eq. (2.1) becomes 


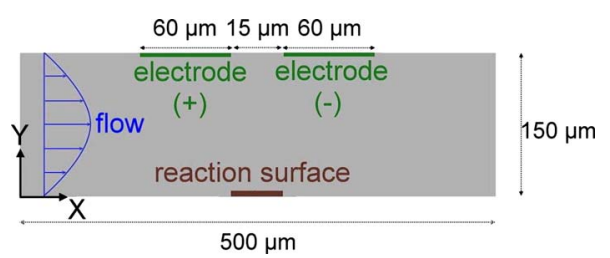

FIG. 1. (Color online) Sketch of the 2D model. Size of reaction surface is $40 \times 3 \mu \mathrm{m}^{2}$.

$$
\begin{aligned}
\overrightarrow{F_{E}}= & -\frac{1}{2} \varepsilon\left[0.024(\nabla T \cdot \vec{E}) \frac{\vec{E}}{1+(\omega \tau)^{2}}\right. \\
& \left.+\frac{|\vec{E}|^{2}}{2}(-0.004) \nabla T\right] .
\end{aligned}
$$

\section{B. The electric field}

Because the electrothermal force is a time-averaged entity, it is sufficient to consider the quasistatic electric field which is the root mean square (rms) value of the ac field. The electrostatic field is related to the electrical potential $\Phi$ by ${ }^{17}$

$$
\vec{E}=-\nabla \Phi,
$$

and $\Phi$ satisfies

$$
\nabla^{2} \Phi=0
$$

where $\Phi$ the electrical potential.

\section{The temperature field}

A small amount of Joule heating could give rise to a temperature increase in the fluid. In order to estimate the temperature rise for a given electrode system, the following energy balance equation must be solved: ${ }^{18}$

$$
\rho c_{p} \frac{\partial T}{\partial t}+\rho c_{p} \vec{V} \cdot \nabla T=k \nabla^{2} T+\sigma|\vec{E}|^{2},
$$

where $\rho, c_{p}, \vec{V}$, and $k$ are the density of the fluid, specific heat, velocity of the fluid, and thermal conductivity of the fluid, respectively. Here $\sigma|\vec{E}|^{2}$ is the Joule heating as a source term.

\section{The flow field}

In this work it is assumed that the fluid is incompressible so that

$$
\frac{\partial u}{\partial x}+\frac{\partial v}{\partial y}=0
$$

where $u$ and $v$ are the $x$ and $y$ velocity components, respectively. The equations of motion are

$$
\rho \frac{\partial u}{\partial t}+\rho\left(u \frac{\partial u}{\partial x}+v \frac{\partial u}{\partial y}\right)-\eta \nabla^{2} u+\frac{\partial p}{\partial x}=F_{E, x},
$$

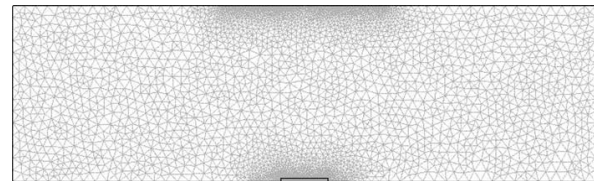

FIG. 2. 2D unstructured mesh with triangular elements.

$$
\rho \frac{\partial v}{\partial t}+\rho\left(u \frac{\partial v}{\partial x}+v \frac{\partial v}{\partial y}\right)-\eta \nabla^{2} v+\frac{\partial p}{\partial y}=F_{E, y},
$$

where $\eta$ is the dynamic viscosity of the fluid and $p$ is the pressure. In this work it is assumed that the density $\rho$ and viscosity $\eta$ of the modeled incompressible fluid are constant independent of temperature and concentration.

\section{E. The concentration field}

Transport of analytes to and from the reaction surface is assumed to be described by Fick's second law with convective terms

$$
\frac{\partial[A]}{\partial t}+u \frac{\partial[A]}{\partial x}+v \frac{\partial[A]}{\partial y}=D\left(\frac{\partial^{2}[A]}{\partial x^{2}}+\frac{\partial^{2}[A]}{\partial y^{2}}\right),
$$

where $[A]$ is the concentration of analyte and $D$ is the diffusion coefficient of analyte.

\section{F. The reaction surface}

The reaction between immobilized ligand and analyte is assumed to follow the first order Langmuir adsorption model. ${ }^{4,19}$ During the reaction, the analyte-ligand complex $[A B]$ increases as a function of time according to the reaction rate

$$
\frac{\partial[A B]}{\partial t}=k_{a}[A]_{\text {surface }}\left\{\left[B_{0}\right]-[A B]\right\}-k_{d}[A B],
$$

where $[A]_{\text {surface }}$ is the concentration of the analyte at the reaction surface by mass-transport, $\left[B_{0}\right]$ is the surface concentration of the ligand, and $[A B]$ is the surface concentration of the analyte-ligand complex, respectively.

\section{SIMULATION DETAILS}

Sketch of the 2D model of the microchannel is shown in Fig. 1. In this work, the dimensions of the reaction surface of biosensor and microchannel are $40 \times 3$ and $500 \times 150 \mu \mathrm{m}^{2}$, respectively. The thickness of electrode is neglected. The buffer solution mixed with the analytes flows from the left to the right. On the reaction surface are the immobilized ligands. A pair of electrodes is put on the top of the microchannel. An ac electric field is applied through the electrodes.

Figure 2 shows the unstructured mesh consisting of triangular elements generated in the calculation. It is noticed that the regions nearby the two electrodes and the reaction surface are refined with a better mesh quality.

\section{A. The electric field configuration}

In an immunoassay experiment, phosphate buffer saline is usually used to be a neutral buffer solution $(p \mathrm{H}=7.2)$, 
which is mixed with analytes as a carrier fluid. So the fluid in the microchannel can be assumed that its physical properties are similar to water. The relative permittivity $\varepsilon_{r}$ and the electrical conductivity $\sigma$ are 80.2 and $5.75 \times 10^{-2} \mathrm{~S} \mathrm{~m}^{-1}$, respectively.

The applied voltage is $15 \mathrm{~V}_{\text {rms }}$ peak-to-peak with an operating frequency of $100 \mathrm{kHz}$. Boundary conditions are $\Phi$ $= \pm V_{\mathrm{rms}} / 2$ at the two electrodes and electrical insulated elsewhere.

\section{B. The temperature field configuration}

The fluid in this domain is assumed to have properties as water. The density, specific heat, and thermal conductivity are $10^{3} \mathrm{~kg} / \mathrm{m}^{3}, 4184 \mathrm{~J} / \mathrm{kg} \mathrm{K}$, and $0.6 \mathrm{~W} / \mathrm{m} \mathrm{K}$, respectively. The temperature at the inlet is maintained constant and the temperature boundary condition at the outlet is set as convective heat flux.

The parts of top wall with the horizontal location coinciding with the two electrodes are kept in constant temperature, say $T=300 \mathrm{~K}$, by using a thermoelectric cooler. Elsewhere on the top wall and the whole bottom wall are kept thermally insulated. It is noted that different settings for the temperature boundary conditions on the top and bottom walls will produce different temperature fields in the reaction channel $^{20}$ and result in different degrees of enhancement of the binding reaction. We will discuss the effect of varying the boundary conditions on the enhancement of the binding reaction in Sec. IV.

\section{The flow field configuration}

The value of dynamic viscosity $\eta$ is set as that of water, $10^{-3} \mathrm{~Pa}$ s. Since the flow in the microchannel is in low Reynolds number condition, it is assumed as a laminar flow. The average velocity of the parabolic profile is set to $u$ $=10^{-4} \mathrm{~m} / \mathrm{s}$ at the inlet. Boundary conditions are $p=0$ at the outlet, and nonslip elsewhere.

\section{The concentration field configuration and kinetics of the specific binding}

The diffusion coefficients of human CRP $(=2.175$ $\left.\times 10^{-11} \mathrm{~m}^{2} / \mathrm{s}\right)$ and $\mathrm{IgG}\left(=5 \times 10^{-11} \mathrm{~m}^{2} / \mathrm{s}\right)$ are obtained from Refs. 21 and 22. The inlet concentration of analyte is chosen as $[A]=6.4 \mathrm{nM}$. The initial surface concentration $\left[B_{0}\right]$ is assumed as $1.4 \times 10^{-8} \mathrm{~mole} / \mathrm{m}^{2}{ }^{23}$

The diffusive flux at the reaction surface should be balanced against the reaction rate

$$
-D\left(\frac{\partial[A]}{\partial y}\right)_{\text {surface }}=k_{a}[A]_{\text {surface }}\left\{\left[B_{0}\right]-[A B]\right\}-k_{d}[A B] .
$$

The association rate constant $k_{a}$ and dissociation rate constant $k_{d}$ of the protein pairs can be found in Ref. 24. The $k_{a}$ and $k_{d}$ for CRP-anti-CRP binding interactions are 1.0 $\times 10^{7} \mathrm{M}^{-1} \mathrm{~s}^{-1}$ and $2.6 \times 10^{-2} \mathrm{~s}^{-1}$, respectively. The $k_{a}$ and $k_{d}$ for IgG-anti-IgG binding interactions are 2.5 $\times 10^{5} \mathrm{M}^{-1} \mathrm{~s}^{-1}$ and $3 \times 10^{-4} \mathrm{~s}^{-1}$, respectively.

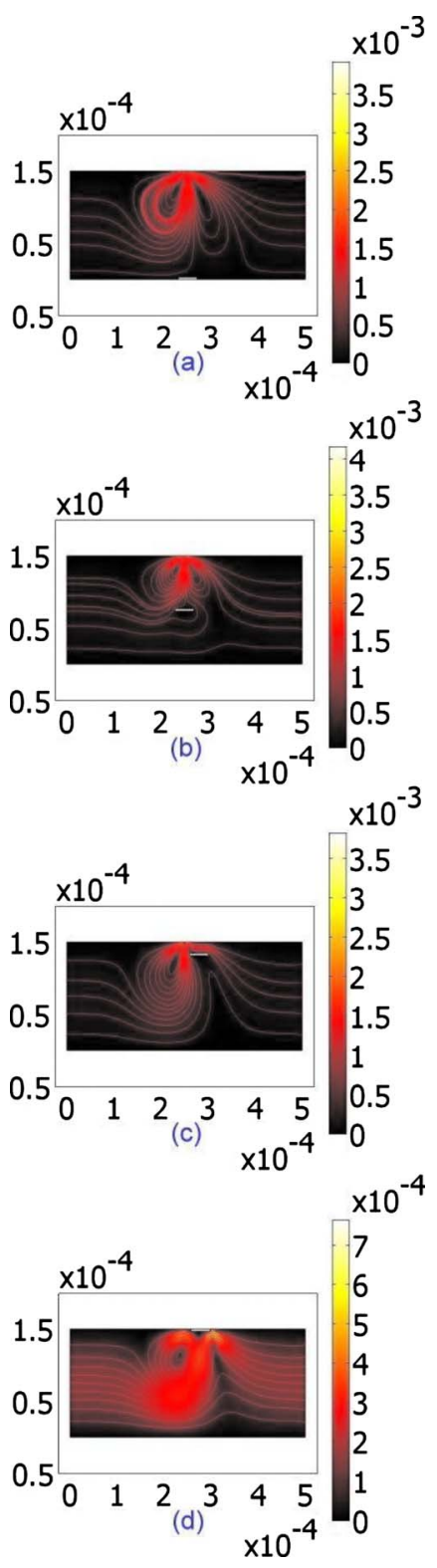

FIG. 3. (Color online) The distribution of the flow velocity field when the applied voltage is $15 \mathrm{~V}$ with different positions of the reaction surface: (a) $(250,1.5)$, (b) $(250,75)$, (c) $(281,133)$, and (d) $(277.5,145.5)$, respectively.

The initial conditions for both the concentrations of the analyte in the bulk, $[A]$, and the concentrations of the analyte-ligand complex on the reaction surface, $[A B]$, are all zero, respectively.

We simulate the dissociation phase of the binding reaction of the two protein pairs by terminating the supply of the analyte at a time after the binding reaction is saturated. The time of discontinuing the analyte is set manually.

\section{RESULTS}

The $2 \mathrm{D}$ simulation to evaluate the binding curves during the association and dissociation with or without an applied voltage has been performed by the finite element analysis software, COMSOL MULTIPHYSICS ${ }^{\mathrm{TM}}$. $^{25}$ The results are examined to be mesh independent by means of a convergence test. 

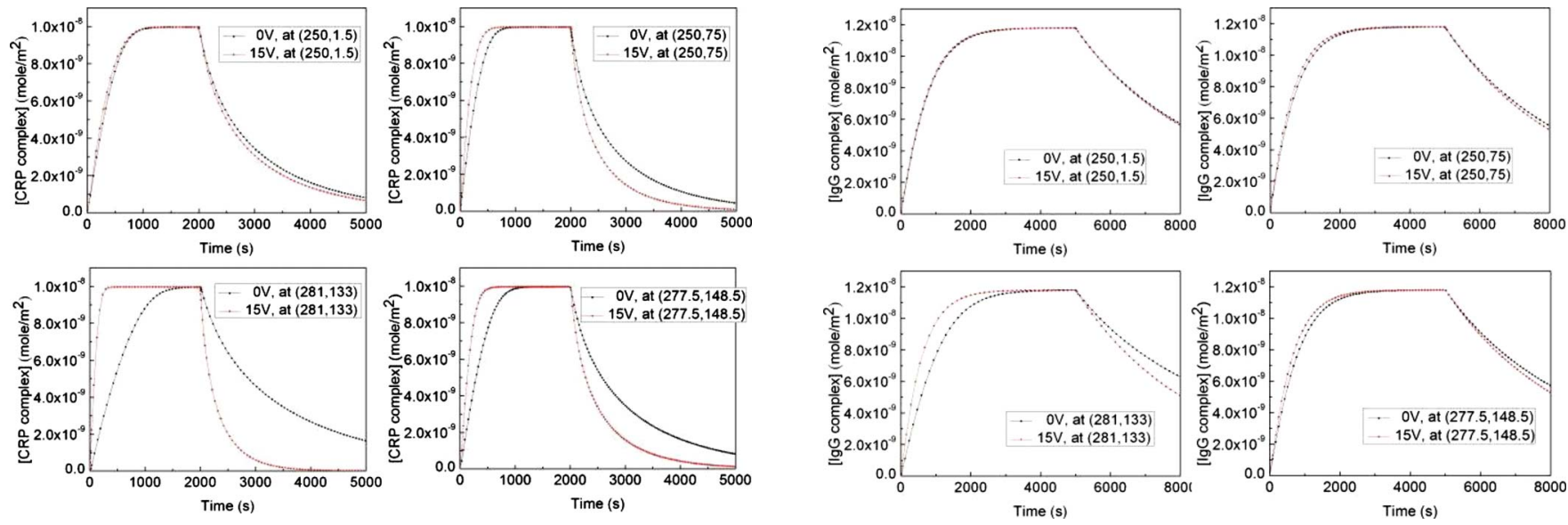

FIG. 4. (Color online) The surface concentration of CRP complex as a function of time with or without applying voltage for the four locations defined in Fig. 3.

FIG. 5. (Color online) The surface concentration of IgG complex as a function of time with or without applying voltage for the four locations defined in Fig. 3.

TABLE I. The initial slopes and enhancement factors of CRP binding reaction.

\begin{tabular}{lcccc}
\hline \hline \multicolumn{1}{c}{ Curve } & $\begin{array}{c}\text { Initial slope } \\
\text { (association) } \times 10^{-11}\end{array}$ & $\begin{array}{c}\text { CRP binding reaction } \\
\text { Initial slope } \\
\text { (dissociation) } \times 10^{-11}\end{array}$ & $\begin{array}{c}\text { Enhancement factor } \\
\text { (association) }\end{array}$ & $\begin{array}{c}\text { Enhancement factor } \\
\text { (dissociation) }\end{array}$ \\
\hline $0 \mathrm{~V},(250,1.5)$ & 1.80 & -1.41 & & \\
$0 \mathrm{~V},(250,75)$ & 2.48 & -1.81 & & \\
$0 \mathrm{~V},(281,133)$ & 1.15 & -0.95 & & \\
$0 \mathrm{~V},(277.5,148.5)$ & 1.80 & -1.39 & 1.18 & 1.14 \\
$15 \mathrm{~V},(250,1.5)$ & 2.13 & -1.61 & 1.62 & 1.48 \\
$15 \mathrm{~V},(250,75)$ & 4.02 & -2.69 & 5.17 & 3.74 \\
$15 \mathrm{~V},(281,133)$ & 5.94 & -3.54 & 2.20 & 1.79 \\
$15 \mathrm{~V},(277.5,148.5)$ & 3.95 & -2.49 & & \\
\hline \hline
\end{tabular}

TABLE II. The initial slopes and enhancement factors of IgG binding reaction.

\begin{tabular}{lcccc}
\hline \hline \multicolumn{1}{c}{ Curve } & $\begin{array}{c}\text { Initial slope } \\
\text { (association) } \times 10^{-11}\end{array}$ & $\begin{array}{c}\text { IgG binding reaction } \\
\text { Initial slope } \\
\text { (dissociation) } \times 10^{-11}\end{array}$ & $\begin{array}{c}\text { Enhancement factor } \\
\text { (association) }\end{array}$ & $\begin{array}{c}\text { Enhancement factor } \\
\text { (dissociation) }\end{array}$ \\
\hline $0 \mathrm{~V},(250,1.5)$ & 1.26 & -2.96 & & \\
$0 \mathrm{~V},(250,75)$ & 1.43 & -3.16 & & \\
$0 \mathrm{~V},(281,133)$ & 0.91 & -2.60 & & \\
$0 \mathrm{~V},(277.5,148.5)$ & 1.25 & -2.94 & 1.06 & 1.03 \\
$15 \mathrm{~V},(250,1.5)$ & 1.34 & -3.05 & 1.14 & 1.04 \\
$15 \mathrm{~V},(250,75)$ & 1.63 & -3.27 & 1.93 & 1.28 \\
$15 \mathrm{~V},(281,133)$ & 1.76 & -3.32 & 1.22 & \\
$15 \mathrm{~V},(277.5,148.5)$ & 1.53 & -3.07 & & \\
\hline \hline
\end{tabular}



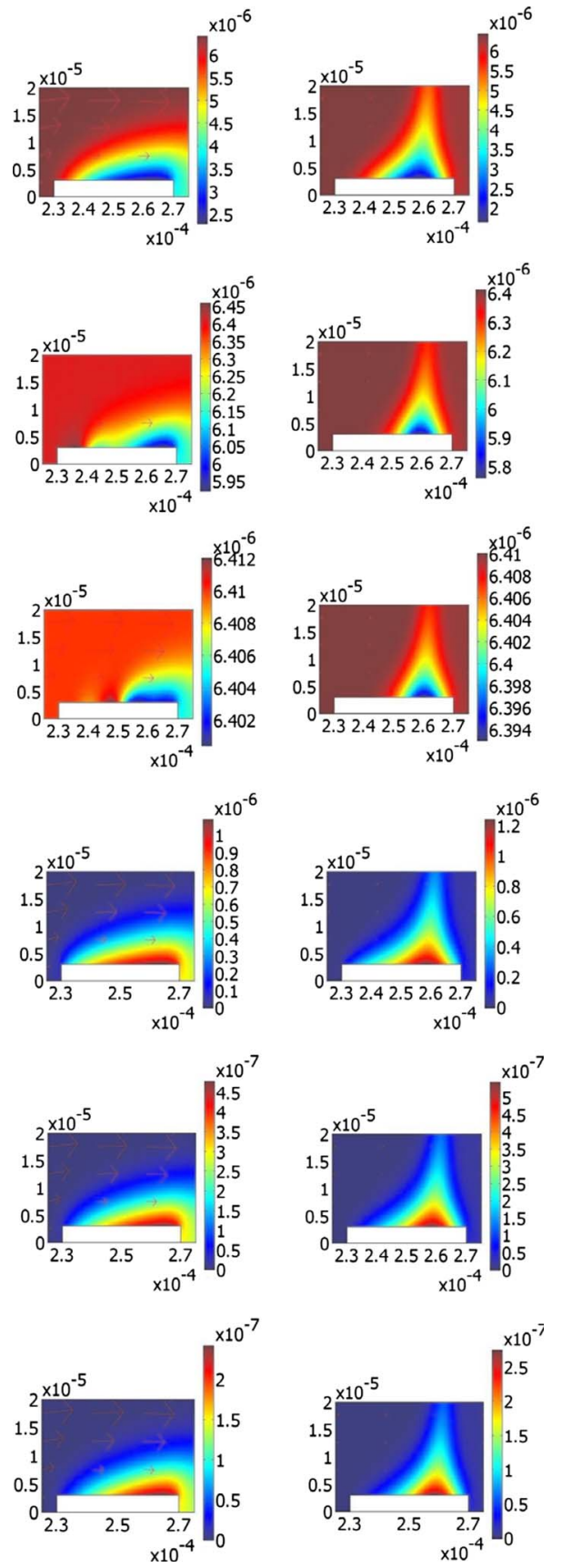

FIG. 6. (Color online) The development of the diffusion boundary layer of the CRP binding reaction without (the left panel) or with (the right panel) applying voltage $15 \mathrm{~V}_{\text {rms }}$. The reaction surface is located at $(250,1.5)$. Notice that for the left or right panel, the first three illustrations are in association phase at the time of 500,1000, and $1500 \mathrm{~s}$, and the last three illustrations are in dissociation phase at the time of 3000, 4000, and $5000 \mathrm{~s}$. Notice that the density scales are different to increase the plot visibility.

\section{A. Stirring vortices and position effect of reacting surface for binding enhancement}

The electrothermally driven stirring flow field would construct a pair of vortices, which can enhance the binding reaction of the protein pair by a strong convection of the analyte. The existence of the reaction surface at different positions would interfere with the flow field, especially the shape of the vortices, to different degrees. This effect influences the binding reaction. An optimal position of the reaction surface can be found to have the largest enhancement.
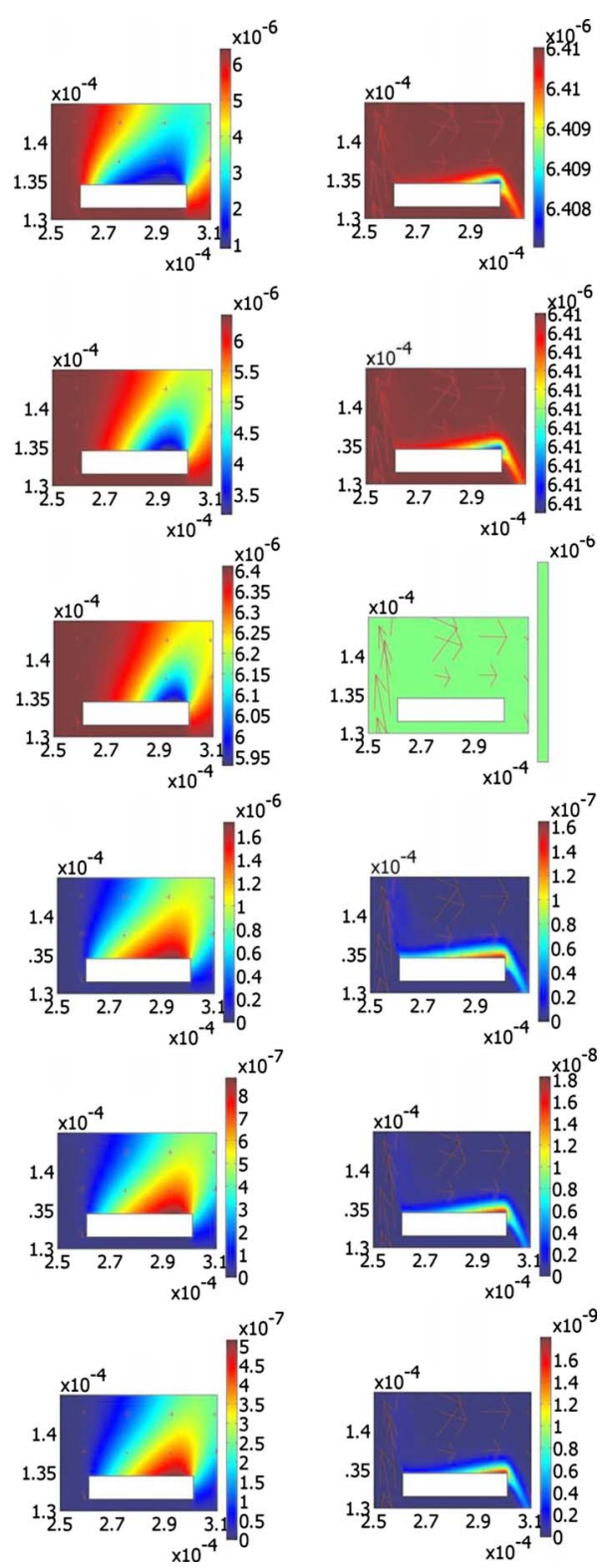

FIG. 7. (Color online) The development of the diffusion boundary layer of the CRP binding reaction without (the left panel) or with (the right panel) applying voltage $15 \mathrm{~V}_{\text {rms. }}$. The reaction surface is located at $(281,133)$. Notice that for the left or right panel, the first three illustrations are in association phase at the time of 500, 1000, and $1500 \mathrm{~s}$, and the last three illustrations are in dissociation phase at the time of 3000, 4000, and 5000 s. Notice that the density scales are different to increase the plot visibility.

Figures 3(a)-3(d) show the flow fields and the vortices induced by the nonuniform electrothermal force, when the applied voltage is $15 \mathrm{~V}_{\text {rms }}$ peak-to-peak with an operating frequency of $100 \mathrm{kHz}$, for four positions of the reacting surface. Notice that the reaction surfaces for Figs. 3(a)-3(c) are faced upward and that for Fig. 3(d) is faced downward. It is interesting to observe that a "squeezing" effect has caused the velocity of the flow between the boundary and the reaction surface to be largely increased when the reaction surface is located near the negative electrode, i.e., the position $(281,133)$, as shown in Fig. 3(c), which is in fact the optimal one selected from 30 studied positions. The largely accelerated flow over the reaction surface causes the efficient transport of analyte to the reaction surface and largely increases the association and dissociation speed. 

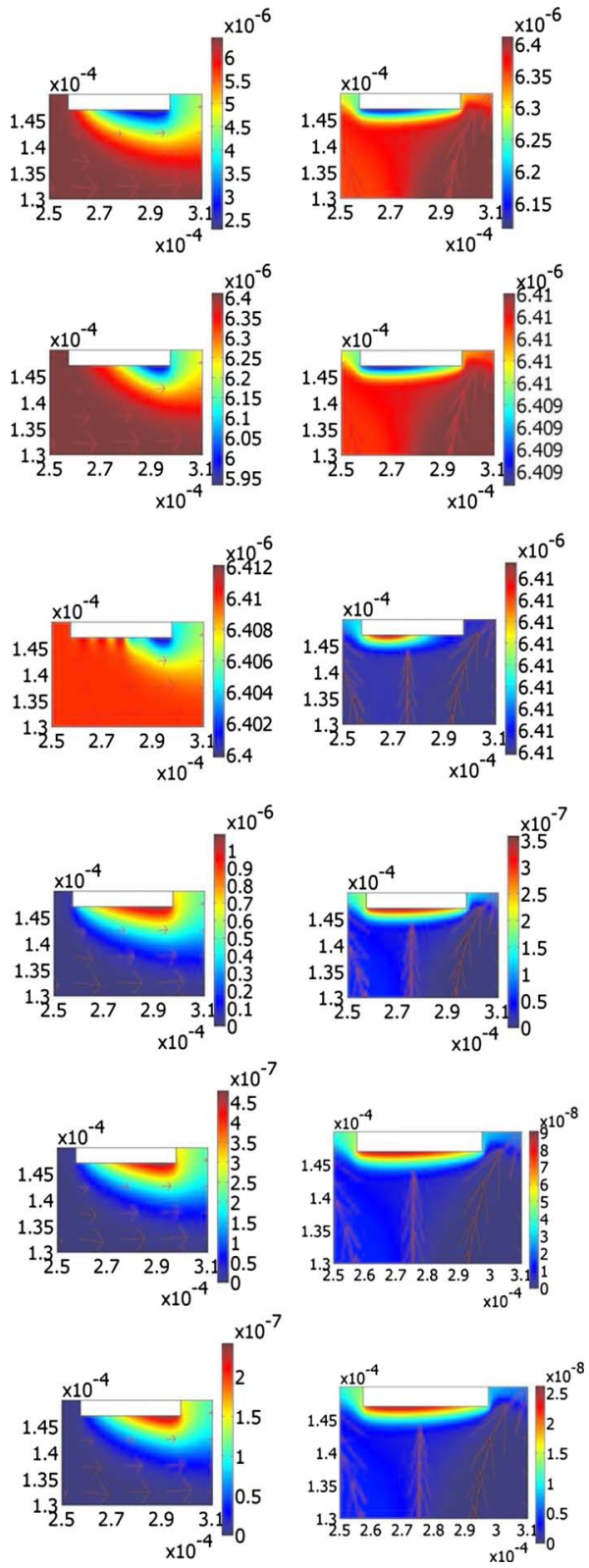

FIG. 8. (Color online) The development of the diffusion boundary layer of the CRP binding reaction without (the left panel) or with (the right panel) applying voltage $15 \mathrm{~V}_{\text {rms }}$. The reaction surface is located at $(277.5,148.5)$. Notice that for the left or right panel, the first three illustrations are in association phase at the time of 500, 1000, and $1500 \mathrm{~s}$, and the last three illustrations are in dissociation phase at the time of 3000, 4000, and $5000 \mathrm{~s}$. Notice that the density scales are different to increase the plot visibility.

In Figs. 4 and 5, the full time scale simulations of the binding reaction curves for $\mathrm{CRP}$ and $\mathrm{IgG}$, corresponding to the configurations shown in Fig. 3, are presented. Consistent results can be observed that the binding reaction for reacting surface located at $(281,133)$ with electrothermal force applied presents the largest reaction speedup. In contrast, the binding reaction for reacting surface located at $(250,1.5)$ without electrothermal force applied has the smallest reaction speedup. In addition, although the dissociation equilibrium constant $\left(K_{D}=k_{d} / k_{a}\right)$ of the two proteins are similar as medium affinity $\left(\mathrm{CRP}: 2.6 \times 10^{-9}\right.$, IgG: $\left.1.2 \times 10^{-9}\right)$, the characteristic behaviors in association phase or dissociation phase are different. The response of CRP is apparently faster
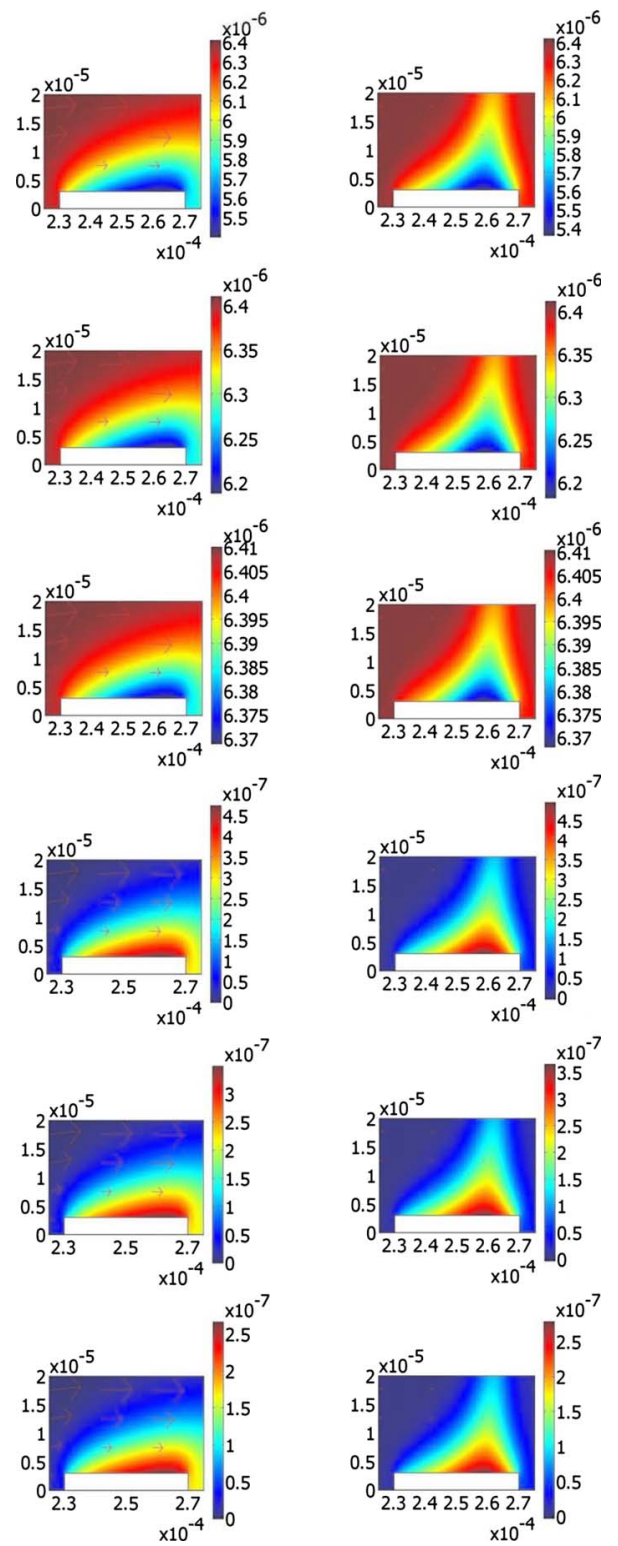

FIG. 9. (Color online) The development of the diffusion boundary layer of the IgG binding reaction without (the left panel) or with (the right panel)

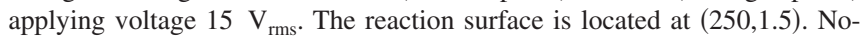
tice that for the left or right panel, the first three illustrations are in association phase at the time of 1000, 2000, and $3000 \mathrm{~s}$, and the last three illustrations are in dissociation phase at the time of 6000,7000 , and $8000 \mathrm{~s}$. Notice that the density scales are different to increase the plot visibility.

than IgG although the magnitudes of their equilibrium constants are similar. The major reason is that both $k_{a}$ and $k_{d}$ of CRP are greater than IgG. This indicates that using electrothermal effects one can separate the responses of different proteins with similar bioaffinity.

Since it is desirable to predict the final saturated concentration of the analyte-ligand complex in a rapid way, the initial slope of the binding curve is a good indicator for biosensor performance. Furthermore, we quantify the enhancement of the association or dissociation of the binding reaction with an enhancement factor. The enhancement factor is defined as the ratio of the initial slope of the binding reaction curve with an applied voltage of $15 \mathrm{~V}_{\text {rms }}$ to the initial slope without applying voltage. Tables I and II show 

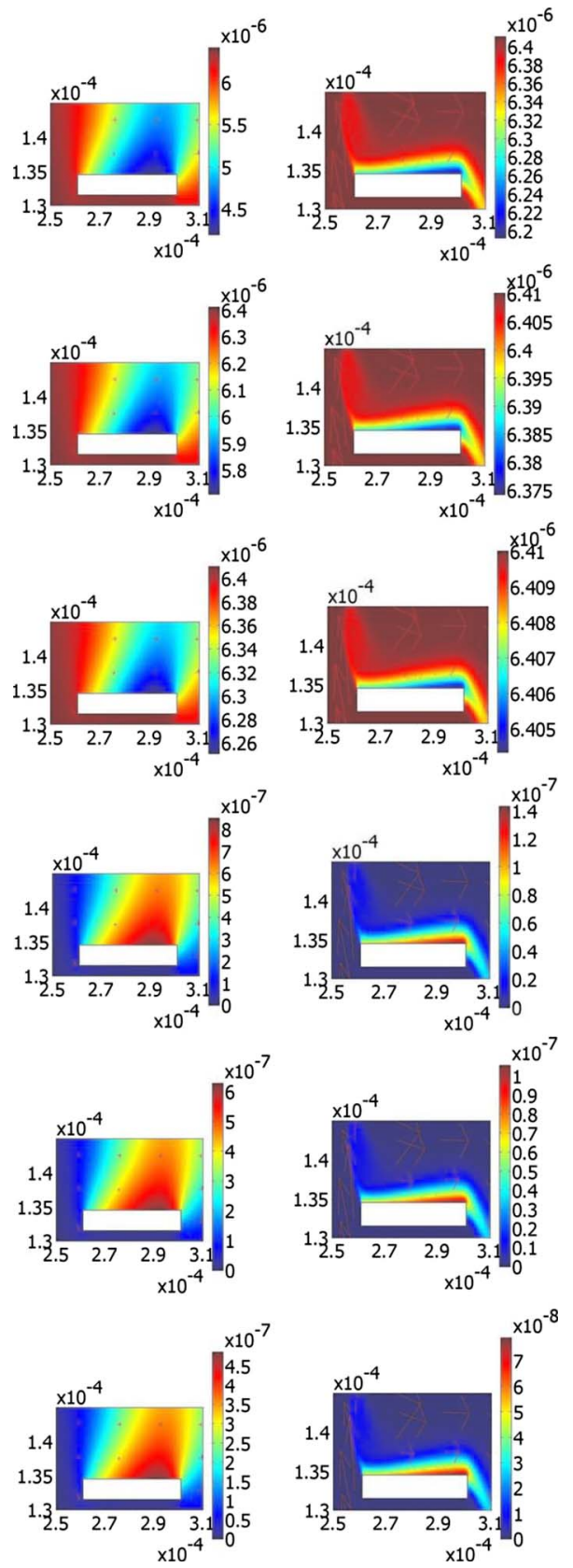

FIG. 10. (Color online) The development of the diffusion boundary layer of the $\mathrm{IgG}$ binding reaction without (the left panel) or with (the right panel) applying voltage $15 \mathrm{~V}_{\text {rms. }}$. The reaction surface is located at $(281,133)$. Notice that for the left or right panel, the first three illustrations are in association phase at the time of 1000,2000 , and $3000 \mathrm{~s}$, and the last three illustrations are in dissociation phase at the time of 6000, 7000, and $8000 \mathrm{~s}$. Notice that the density scales are different to increase the plot visibility.

the initial slopes and the enhancement factors in association and dissociation for CRP and IgG protein pairs, respectively. It is found that the enhancement is higher in the association of the binding reaction than dissociation. The largest enhancement factor is 5.17 in the association of CRP protein pair. In contrast, the IgG protein pair is less sensitive to the effect of electrothermal stirring field since the enhancement factor is less than 2 .

\section{B. The diffusion boundary layer}

Since the reaction surface of the biosensor is a nonslip boundary in the flow field, the diffusion boundary layer can
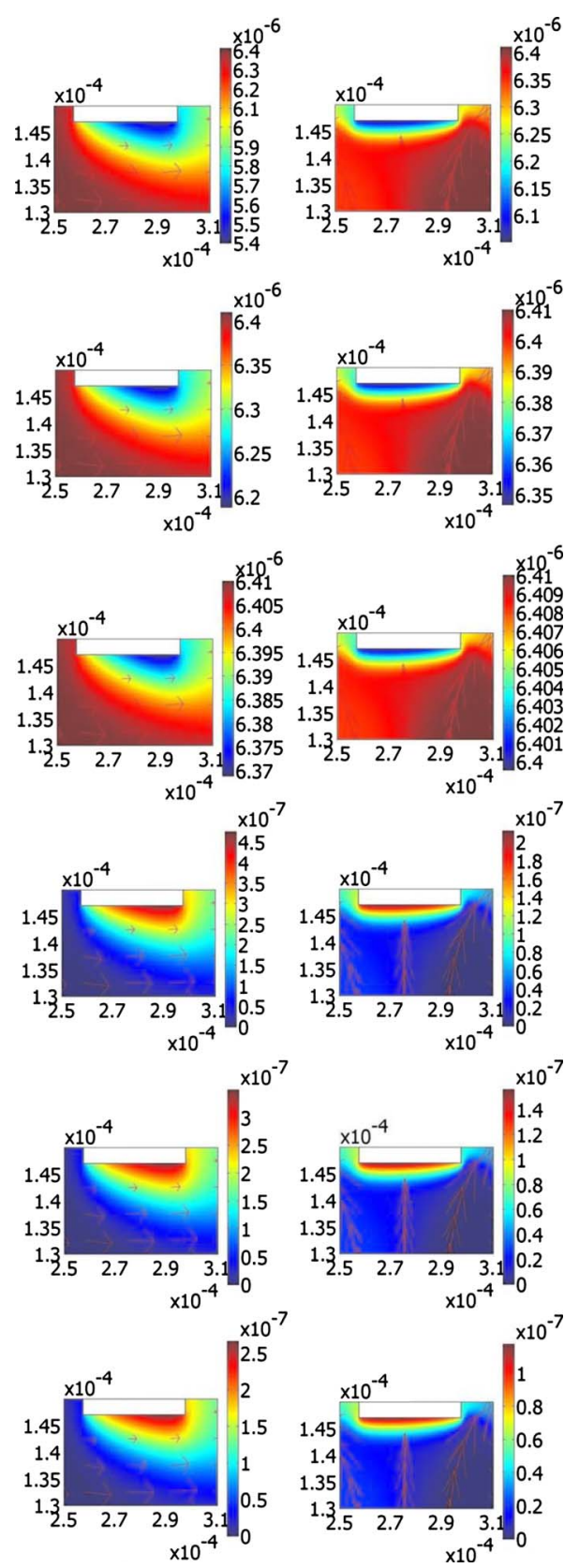

FIG. 11. (Color online) The development of the diffusion boundary layer of the $\mathrm{IgG}$ binding reaction without (the left panel) or with (the right panel) applying voltage $15 \mathrm{~V}_{\mathrm{rms}}$. The reaction surface is located at $(277.5,148.5)$. Notice that for the left or right panel, the first three illustrations are in association phase at the time of 1000, 2000, and $3000 \mathrm{~s}$, and the last three illustrations are in dissociation phase at the time of 6000, 7000, and $8000 \mathrm{~s}$. Notice that the density scales are different to increase the plot visibility.

be easily formed in a laminar flow configuration. Figures 6-8 show the variations of diffusion boundary layer of the CRP protein pairs from the association phase to the dissociation phase without/with applying electrothermal force for the reaction surface at the position $(250,1.5),(281,133)$, and (277.5,148.5), respectively. Instead, Figs. 9-11 are for IgG protein pairs. The left and right panels of these figures are for the cases of without and with electrothermal force applied, respectively. The top three subfigures are for association phase, and the bottom three subfigures are for dissociation phase, in each panel. In Figs. 6 and 9, where the reaction 


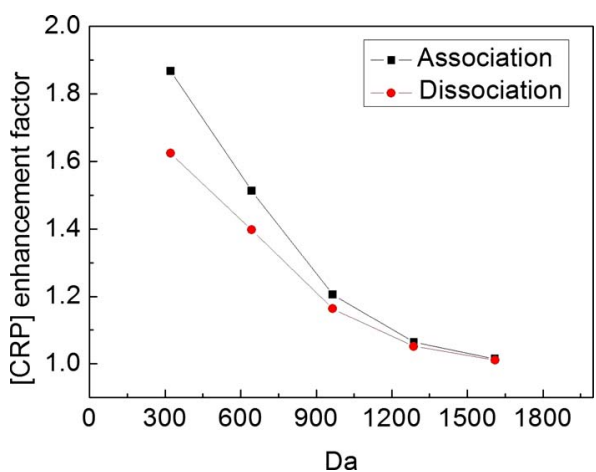

FIG. 12. (Color online) The enhancement factor of CRP binding reaction as a function of $D_{a}$.

surface is located at the bottom surface of the microchannel and opposite to the electrode pairs, the shapes of the diffusion boundary layer are distorted consistently with the flow field shown in the first subfigure of Fig. 3 with the electrothermal force applied. However since the vortices are least strong at this position, the sizes of the diffusion boundary do not change a lot, as expected, and the binding reaction is least enhanced relative to the other positions, as revealed in Figs. 4 and 5.

In Figs. 7 and 10, where the reaction surface is located at the position $(281,133)$, the diffusion boundary layer are suppressed very much and in fact almost eliminated as shown in the third subfigure (at the time of $1500 \mathrm{~s}$ ) of the right panel of Fig. 7, when the induced vortices "squeeze" the flow to pass rapidly through the reacting surface. In the association phase, the flow transports sufficient analyte molecules to the reacting surface. In addition, the gap between the reacting surface and the top surface of the microchannel is relatively much smaller and so it is easier for the analyte molecules to diffuse to the reacting surface. In the dissociation phase, the dissociated analyte molecules are also carried away quickly, which again limits the growth of diffusion boundary layer for dissociation. By efficiently suppressing the growth of the diffusion boundary layers in both phases of association and dissociation, the electrothermal force is truly an effective way to enhance the binding reaction, as revealed in Figs. 4 and 5 .

\section{Effect of the height of microchannel}

The Damköhler number $D_{a}$ is the ratio of reaction velocity $\left(k_{a}\left[B_{0}\right]\right)$ to diffusion velocity $(D / h)$. In this section we fix

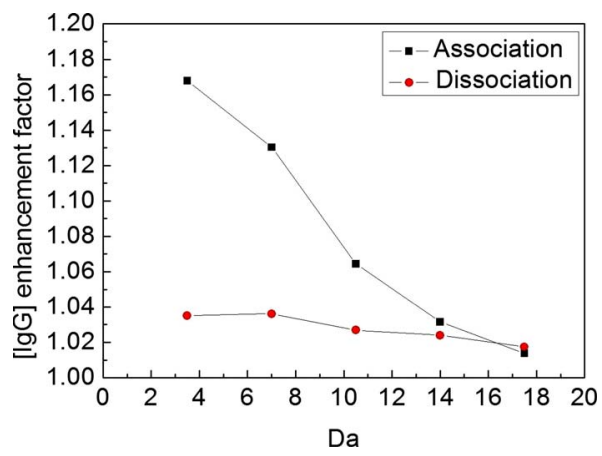

FIG. 13. (Color online) The enhancement factor of $\mathrm{IgG}$ binding reaction as a function of $D_{a}$.
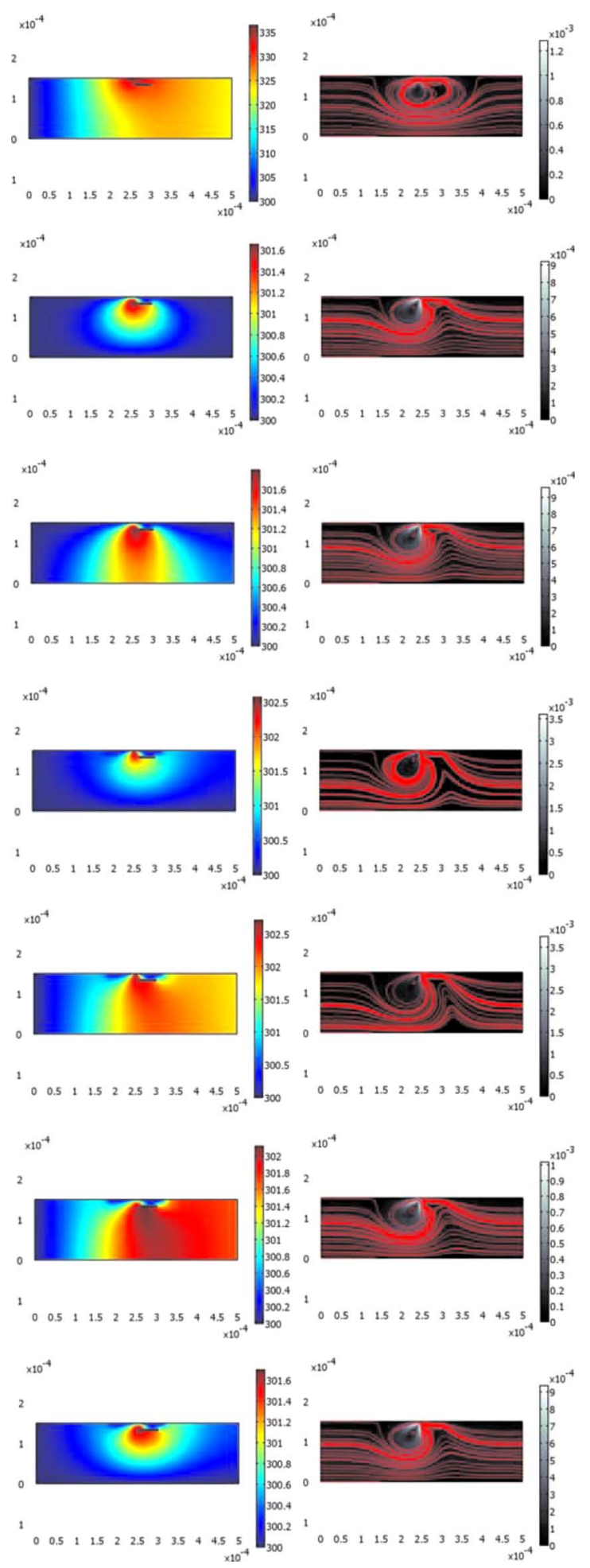

FIG. 14. (Color online) Temperature (left panel) and velocity (right panel) fields in the reaction channel for various temperature conditions of the top and bottom walls. For the left or right panel, the seven illustrations from top to the bottom in descending order correspond to the seven cases of different temperature boundary conditions, namely, cases $1-7$, as described in Sec. IV.

the inlet velocity $(100 \mu \mathrm{m} / \mathrm{s}), k_{a}\left[B_{0}\right]$, and $D$ to investigate how the height of channel affects the enhancement factor. It is noted that in each channel the flow rate is varied but the average velocity is kept to be constant. In addition, the reaction surface is set at the position $(250,1.5)$. Figures 12 and 
TABLE III. The initial slopes and enhancement factors of CRP binding reaction for different temperature boundary conditions.

\begin{tabular}{ccccc}
\hline \hline Curve & $\begin{array}{c}\text { Initial slope } \\
\text { (association) } \times 10^{-11}\end{array}$ & $\begin{array}{c}\text { CRP binding reaction } \\
\text { Initial slope }\end{array}$ & $\begin{array}{c}\text { Enhancement factor } \\
\text { (dissociation) } \times 10^{-11}\end{array}$ & $\begin{array}{c}\text { Enhancement factor } \\
\text { (dissociation) }\end{array}$ \\
\hline Case 1 & 2.05 & -1.39 & 1.78 & 1.47 \\
Case 2 & 4.56 & -2.89 & 3.97 & 3.06 \\
Case 3 & 4.62 & -2.92 & 4.02 & 3.09 \\
Case 4 & 5.82 & -3.49 & 5.07 & 3.69 \\
Case 5 & 5.90 & -3.52 & 5.17 & 3.74 \\
Case 6 & 4.64 & -2.93 & 4.04 & 3.10 \\
Case 7 & 4.55 & -2.89 & 3.96 & 3.05 \\
\hline \hline
\end{tabular}

13, for the CRP and IgG binding reaction curves, respectively, show the enhancement factors with different $D_{a}$ numbers, which is only related with the height of channel. The height of channel varies from 50 to $250 \mu \mathrm{m}$. There is a higher enhancement factor for lower $D_{a}$ numbers (lower channel height) for both CRP and IgG protein pairs. The reason for this effect is that the time required for diffusing the analyte to the reaction surface is shorter with a lower height of channel.

\section{Effect of temperature boundary conditions}

The stirred electrothermal flows generated in the reaction channel are driven by the inhomogeneous temperature field. As noted in Ref. 20 the temperature boundary conditions significantly influence the temperature field and hence could result in a stronger or weaker enhancement depending on the choice of temperature boundary conditions. In this subsection, we consider six additional temperature boundary conditions on the top and bottom walls. However, the boundary conditions of the flow inlet and outlet of the channel remain unchanged, namely, constant temperature at the flow inlet and convective heat flux condition at the flow outlet. It is noted that the center of reaction surface is located at $(281,133)$, which is the optimal position found above with the temperature boundary conditions described in Sec. III. In addition, only the case of CRP-anti-CRP sample is considered here.

These seven cases of different settings of temperature boundary conditions (including the one used above) are listed as follows:

- case 1: top and bottom walls are both thermally insulated;

- case 2: top and bottom walls are both maintained in constant temperature $300 \mathrm{~K}$;

- case 3: top wall is kept in constant temperature $300 \mathrm{~K}$, but bottom wall is thermally insulated;

- case 4: bottom wall and the parts of top wall with the same horizontal location as the two electrodes are kept in constant temperature $300 \mathrm{~K}$ and the rest parts of top wall are thermally insulated;

- case 5: the parts of top wall with the same horizontal location as the two electrodes are kept in constant temperature $300 \mathrm{~K}$, and the rest of top wall and the bottom wall are thermally insulated (this is the setting used in the above simulation);

- case 6: the parts of top wall from the left end of the left electrode to the right end of the right electrode and the whole bottom wall are kept in constant temperature $300 \mathrm{~K}$, and the rest parts of the top wall are thermally insulated;

- case 7: the parts of top wall from the left end of the left electrode to the right end of the right electrode are kept in constant temperature $300 \mathrm{~K}$, and the rest parts of top wall and the whole bottom wall are thermally insulated.

The numerical results for the seven cases are summarized in Fig. 14 and Table III. Figure 14 presents the temperature (left panel) and flow velocity (right panel) fields for each of these cases. Table III compares the enhancement factors among these cases. As shown in Fig. 14, case 1 (insulated on both top and bottom walls) has a much hotter spot near the electrodes (the local temperature rises up to $36 \mathrm{~K}$ ) than those of all the other six cases (the local temperature rises only $1.7-2.7 \mathrm{~K}$ ). It also exhibits a fainter vortex pattern that results in a much smaller binding enhancement factor than those of the other six cases (see Table III). It is also observed that the specific boundary temperature condition (case 5) yields the best binding enhancement. Therefore it is useful to employ the settings of boundary condition to achieve a better design of biosensors.

\section{E. The design of the microchannel}

In the process of fabricating the reacting chamber, the polydimethyl-siloxane is usually used for its high manufacturability and transparency. Since the reaction rate is highly related to the height of the channel, one can design the channel with a neck region near the reaction surface to achieve a time-saving immunoassay. Figure 15 demonstrates the necking design of the microchannel. The height of the neck region, $h_{2}$, varies from30, 90, up to $150 \mu \mathrm{m}$. In addition, the

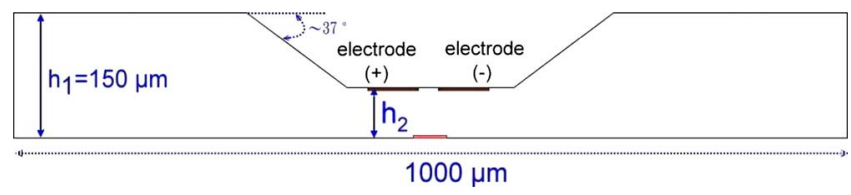

FIG. 15. (Color online) The neck design of the microchannel. 


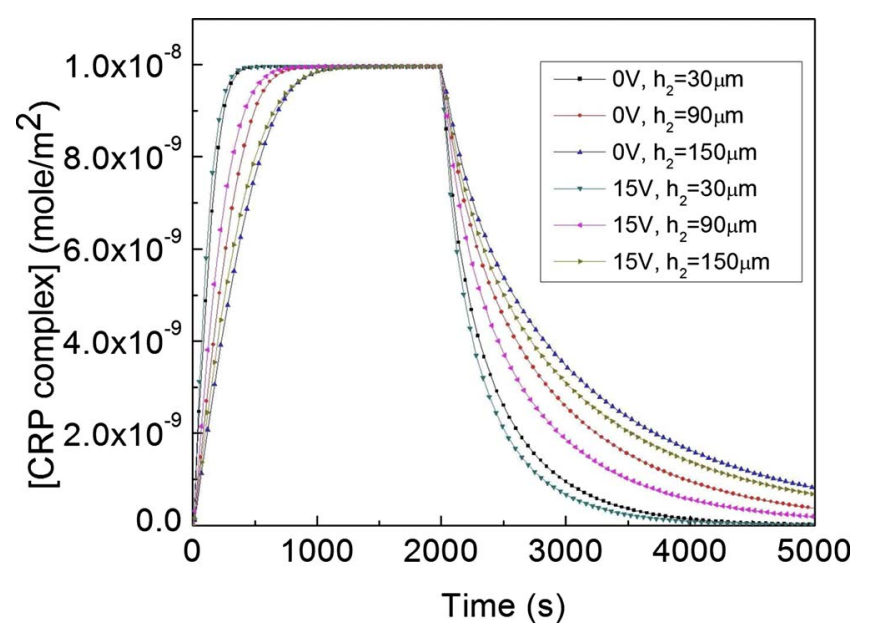

FIG. 16. (Color online) The surface concentration of CRP complex as a function of time with or without applying voltage for various heights of the neck design.

reaction surface is $40 \mu \mathrm{m}$ wide and fixed at the bottom of the channel. The results for CRP binding curves are shown in Fig. 16. It is found that not only the neck region in terms of fast diffusion and convective velocities but also the electrothermally driven vortices (the squeezing effect as discussed before) enhance the binding reaction. With the existence of the stirring flow field, the association rate of the $30 \mu \mathrm{m}$ neck leads the original channel (no neck) by a factor of 2.73 . Combining both the passive mechanisms by changing geometries of the channel shape and the active mechanisms by the electrothermal effect, it is promising that one can achieve the best biosensor design.

\section{CONCLUSION}

In this work a $2 \mathrm{D}$ simulation on the immunoassay in a biosensor with electrothermal effect is performed by the finite element analysis software, COMSOL MULTIPHYSICS ${ }^{\mathrm{TM}}$. Two commonly used proteins, CRP and $\mathrm{IgG}$, are regarded as the analytes for the analysis of the binding kinetics.

A pair of vortices is induced from the electrothermal field to stir the flow and enhance the association and dissociation of the protein pair. Existence of the reaction surface would interfere with the flow field and could change the shape of the vortices. This effect actively enhances the binding reaction. An optimal position of the reaction surface is found to be located at $(281,133)$, yielding the largest enhancement using the temperature boundary conditions described in Sec. III. The enhancement for association is more efficient (up to 5.17 for CRP pair, 3.74 for IgG pair), but less sensitive for dissociation (up to 1.93 for CRP pair, 1.28 for IgG pair). By fixing the sensor locating at $(281,133)$, we also studied the effect of varying the temperature boundary condition on binding enhancement using six additional settings of temperature boundary conditions on the top and bottom walls, including the two limiting cases, namely, constant temperature and thermal insulation. It is found that the temperature boundary conditions do have an essential effect on the enhancement of the binding reaction when applying the thermal electric forces and can be employed to enhance the binding efficiency. A specific setting of the temperature boundary conditions (case 5) yields the best binding enhancement. These results can serve as a useful reference for the design of biosensors.

The analysis for the channel with a neck region is demonstrated for its capability in shortening the required time to reach a steady state. The passive design (neck region) and the active enhancement (electrothermally driven stirring flow) need further experiments to optimize the performance of the system.

\section{ACKNOWLEDGMENTS}

This research was supported by the National Science Council in Taiwan through NSC 96-2120-M-002-014. We thank the NCHC in Taiwan for providing computing resources.

${ }^{1}$ W. S. Tillett and T. Francis, J. Exp. Med. 52, 561 (1930).

${ }^{2}$ N. Camillone, Langmuir 20, 1199 (2004).

${ }^{3}$ W. M. Deen, Analysis of Transport Phenomena (Oxford University Press, New York, 1998).

${ }^{4}$ D. B. Hibbert and J. J. Gooding, Langmuir 18, 1770 (2002).

${ }^{5}$ M. Sigurdson, D. Wang, and C. D. Meinhart, Lab Chip 5, 1366 (2005).

${ }^{6}$ C. K. Yang, J. S. Chang, S. D. Chao, and K. C. Wu, Appl. Phys. Lett. 91, 113904 (2007).

${ }^{7}$ R. Pethig, Crit. Rev. Biotechnol. 16, 331 (1996).

${ }^{8}$ X.-B. Wang, Y. Huang, P. R. C. Gascoyne, and F. F. Becker, IEEE Trans. Ind. Appl. 33, 660 (1997).

${ }^{9}$ H. Morgan, M. P. Hughes, and N. G. Green, Biophys. J. 77, 516 (1999).

${ }^{10}$ A. Ramos, H. Morgan, A. Castellanos, and A. C. Electrokinetics, J. Phys. D 31, 2338 (1998).

${ }^{11}$ N. Green, A. Ramos, A. Gonzalez, H. Morgan, and A. Castellanos, Phys. Rev. 61-4, 4011 (2000).

${ }^{12}$ A. Ramos, A. Gonzalez, N. Green, A. Castellanos, and H. Morgan, Phys. Rev. 61-4, 4019 (2000).

${ }^{13}$ C. Meinhart, D. Wang, and K. Turner, Biomed. Microdevices 5, 139 (2003)

${ }^{14}$ M. Washizu and S. Suzuki, IEEE Trans. Ind. Appl. 30, 835 (1994).

${ }^{15}$ D. Wang, M. Sigurdson, and C. D. Meinhart, Exp. Fluids 38, 1 (2005).

${ }^{16}$ D. R. Lide, CRC Handbook of Chemistry and Physics, 81st ed. (CRC, New York, 2000).

${ }^{17}$ J. A. Stratton, Electromagnetic Theory (McGraw-Hill, New York, 1941).

${ }^{18}$ L. D. Landau and E. M. Lifshitz, Fluid Mechanics (Pergamon, Oxford, 1959).

${ }^{19}$ I. Langmuir, J. Am. Chem. Soc. 40, 1361 (1918).

${ }^{20}$ N. G. Green, A. Ramos, A. González, A. Castellanos, and H. Morgan, J. Electrost. 53, 71 (2001).

${ }^{21}$ Y. Hokama, M. K. Coleman, and R. F. Riley, J. Immunol. 95, 156 (1965).

${ }^{22}$ H. A. Leddy and F. Guilak, Ann. Biomed. Eng. 31, 753 (2003).

${ }^{23}$ E. Behravesh, V. I. Sikavitsas, and A. G. Mikos, Biomaterials 24, 4365 (2003).

${ }^{24}$ C. Chou, H. Y. Hsu, H. T. Wu, K. Y. Tseng, A. Chiou, C. J. Yu, Z. Y. Lee, and T. S. Chan, J. Biomed. Opt. 12, 024025 (2007).

${ }^{25}$ COMSOL Multiphysics, Version 3.3, COMSOL Ltd., Stokhelm. 